\title{
Texture Segmentation by Grouping Ellipse Ensembles via Active Contours
}

\author{
Gui-Song Xia \\ xia@enst.fr
}

Fei Yuan

fei.yuan.nlpr@gmail.com

\author{
CNRS LTCI, Telecom ParisTech \\ 46 rue Barrault, 75013 \\ Paris, France
}

NLPR/LIAMA, Institute of Automation

CAS, Beijing, China

\begin{abstract}
We address the problem of unsupervised segmentation of textural images by relying on morphological image representations and active contours. More precisely, starting with the assumption that textures are statistical ensembles of local image structures, known as textons, we first suggest to represent a texture image through a tree of ellipses, which are derived from the level lines of the image; we then investigate the statistical properties on the tree of ellipses and finally achieve a segmentation of the texture image by grouping all the ellipses into several subsets according to some statistical measurements. The grouping process is formulated as an energy minimizing problem and the solution is obtained by evolving an active contour based on Kullback-Leibler (KL) divergence through a fast global minimization method. Thanks to the proposed ellipsebased features, the segmentation method can integrate local and global information in the image. The experiments on both synthesized and natural texture images validate the approach.
\end{abstract}

\section{Introduction}

Texture plays an important role in human visual perception and offers crucial cues for solving a wide range of computer vision problems, such as image segmentation or scene analysis. The segmentation of texture is a key problem in computer vision and image understanding, the objective of which is to partition an image into several regions characterized by homogeneous texture attributes. Over the course of the past 40 years, numerous studies have been performed for texture segmentation, see $[\because, \square, \square, \square, \square, \square]$. In this paper, we address the issue of structured texture segmentation, starting with the assumption that textures are statistical ensembles of local image structures, also known as textons [ $\square, \mathbf{\square}]$.

In the literatures, many models have been proposed to analyze and segment textures by using structured approaches. Among those, the early work of Beck et al. [ $[$ ] argued that textural segmentation occurs on the basis of the distribution of simple properties, such as brightness, color, size, the slopes of contours and lines of the elemental descriptors, of "texture elements". Julesz [ $\square$ ] proposed to use textons, a set of empirical texture features including elongated blobs, line ending or terminators, for computational texture modeling, and the segmentation of texture was consequently achieved through these features. In [ $\square]$, 
Zhu et al. provided a computational model of textons by relying on Gabor base functions and a specified vocabulary of texton templates. Recently, Todorovic et al. [ $\square, \square]$ suggested to extract explicit textural elements from hierarchical segmentation tree and made use of them for texture segmentation. These approaches provide impressive segmentation results on highly structured texture images. However, one main disadvantage of such methods is that the computation or detection of such textons is not trivial itself. Moreover, modeling the interactions between textons may involve heavy computation.

The study presented in this paper is inspired by works in mathematic morphology, more precisely, by granulometry [ $[\mathbf{8}, \mathbb{0}]$, which characterize textures relying on responses to morphological filtering with user-specified structuring elements of increasing size. The segmentation of synthetic and simple textural images can be achieved by partitioning the image according to some statistics of the granulometry [س, $\square, \square]$, but it fails at describing complicated and highly structured textures [ $\square]$. Instead of using structuring elements and improving the discriminative powerful, alternative approaches have been proposed to analyze textures based on connected operators which perform directly on the level lines of images, see $[\square, \mathbb{\square}, \mathbb{\square}]$. The main motivation of this paper is to investigate the granulometry-like approach in the context of texture segmentation.

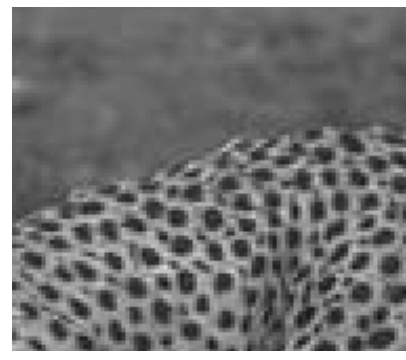

(a)

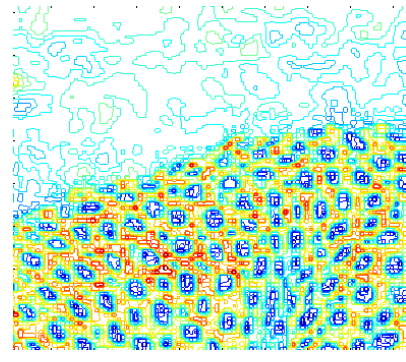

(b)

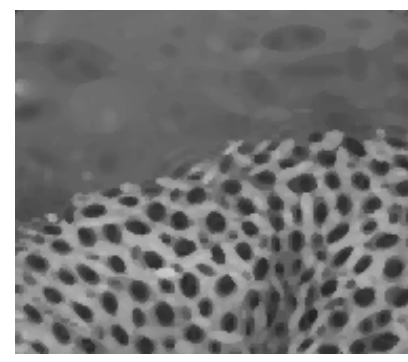

(c)

Figure 1: Represent an image by a tree of ellipse. (a) an original texture image; (b) a subset of its level lines; (c) the image reconstructed from the tree of ellipses, where each shape, i.e. the interior of a level line, is replaced by an ellipse.

Along the line of textons, we first suggest to represent textures by a tree of ellipses, which are derived from the level lines of images and can be regarded as explicit textons, see Figure 1 for a preliminary graphical illustration. Observe that the ellipse-based representation in Figure 1(c) has very similar visual appearance to the original image. As we shall see in the following sections, the tree of ellipses of an image can be computed rapidly and efficiently, thus the proposed approach can overcome difficulties in the detection of texture primitives or texture elements as encontered in [ $[\mathbf{\square}, \mathbb{Z}]$. Based on this representation, textures are subsequently characterized by geometric properties of and by relationships between these ellipses. Texture segmentation is performed by grouping these properties in a unsupervised way. Specifically, the grouping step benefits from an active contour model based on Kullback-Leibler (KL)-divergence similar to the one of [ㅁ] ].

The contribution of this paper is to propose a new texture segmentation approach by relying on an ellipse-based texture representation, where ellipses are regarded as texture elements. We argue that natural texture images can be approximated well by a tree of ellipses and the boundaries between two texture regions can be identified by grouping these ellipse ensembles according to some statistical properties with an active contour model. This work 
somehow fills the gap between granulometry and texton theory on segmentation. Thanks to the ellipse-based features, which might be non-local, the segmentation method can integrate local and global information in the image. Furthermore, the proposed approach is flexible: it allows to segment images subjected to scaling and rotations and it is robust to illumination changes inside the image. In this paper, we also adapts the KL-divergence based active contour model to multi-features, which enables us to take into account different texture cues for segmentation.

The remainder of the paper is organized as follows. In Section 2, we present the ellipsebased texture representation. We then describe in detail the unsupervised texture segmentation with active contours in Section 3. In Section 4, we provide experimental results and finally conclude the paper in Section 5.

\section{Texture representation by a tree of ellipses}

In this section, we first recall the basics of the topographic map representation of images and then describe the ellipse-based texture representation

\subsection{Topographic map of an image: a tree of shapes}

The topographic map representation of images has been first introduced by Caselles et al. [0] in computer vision. It is a hierarchical structure composed of shapes and relying on connected components of level sets. It is a powerful way to represent the geometrical content of an image [曰, 由, 四].

For a gray-scale image $u: \Omega \mapsto R$, the upper and lower level sets are defined respectively as $\chi_{\lambda}(u)=\{x \in \Omega ; u(x) \geq \lambda\}$ and $\chi^{\lambda}(u)=\{x \in \Omega ; u(x) \leq \lambda\}$, for $\lambda \in R$. The topographic map of the image $u$ is made of the connected components of the topological boundaries of the upper level sets (equivalently of lower level sets) of the image. Both the connected components of upper level sets and those of the lower level sets are embedded in a tree structure. These two tree structures are redundant and can be combined into a single one, by drawing on the notion of shape defined as the interior of a level line, i.e. the boundary of a level set. An efficient way to compute the tree of shapes of images is developed in [ष] , named fast level set transformation (FLST). (The codes of FLST can be downloaded at http://megawave.cmla.ens-cachan.fr/.) Figure 2(a) and 2(b) respectively show the topographic map of a synthetic image and a real image.

The tree of shapes of an image has many interesting properties: (1) it yields a scale space without any geometrical degradation; (2) it is a complete image representation (the image can be reconstructed from it) that encodes both the geometric and radiometric information simultaneously; (3) it is also invariant to any local contrast changes, as defined in [ $\mathrm{\Xi}]$.

\subsection{Texture modeling through the tree of ellipses}

Figure 1(b) suggests that topographic maps reflect the structures of texture. This observation has first been addressed in [ $[0]$ and has been widely used as a basic assumption by followings $[\square, \mathbb{\square}]$ on texture analysis relying on topographic maps.

Here, we suggested that, indeed, textures can be well approximated and represented by using a tree of ellipses. It implies that each shape on the topographic map is replaced by an ellipse with the same second-order moments. In Figure 3, we show the ellipse-based 

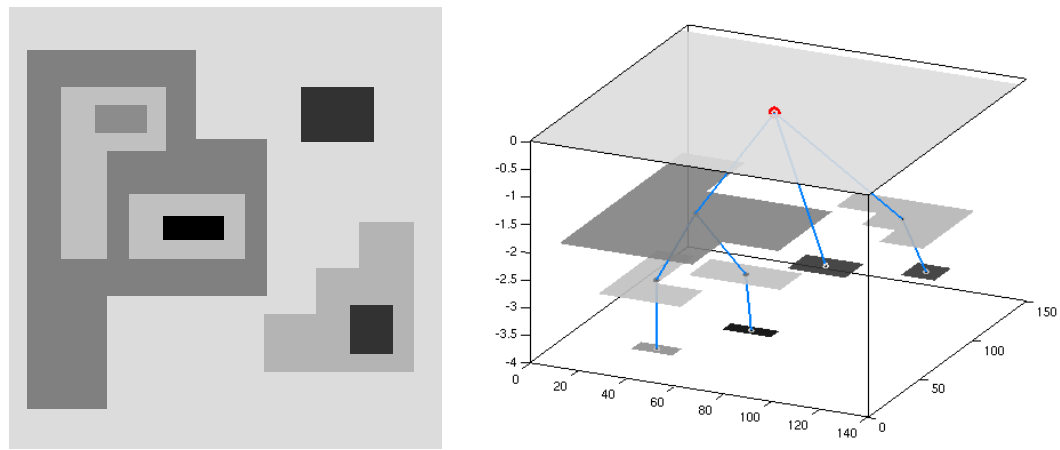

(a) The tree of shapes representation (right) of a synthetic image (left).
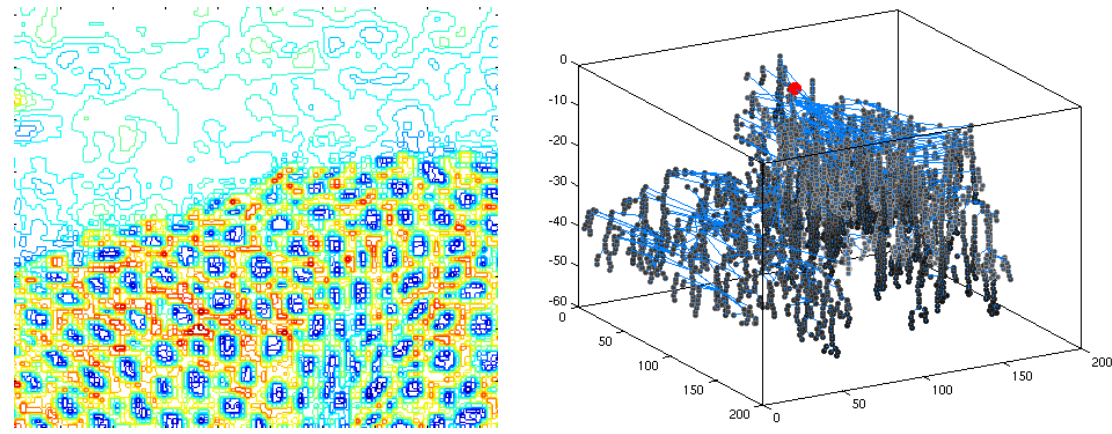

(b) The tree of shapes representation of the texture image in Figure 1. Left: the shapes displayed by their boundaries; Right: the tree structure, i.e. relationships between shapes.

Figure 2: The tree of shapes representation of images. In order to show the tree structure, in the left graphs of (b), shapes are indicated by points and the parent-child relationships between two shapes are expressed by line segments. The root of the tree is the image frame, denoted by a small circle in red.

texture representation of several natural textures ${ }^{1}$, observing how textures are approximated by ellipses. These textures, including textures with elongated structures (the textures in the middle of Figure 3), appear to be well described by a tree of ellipses.

Thus, the modeling of a texture $u$ is reduced to the modeling of the tree of ellipses $(\mathcal{E}, \mathcal{T})$, as

$$
p(u)=p(\mathcal{S}, \mathcal{T}) \approx p(\mathcal{E}, \mathcal{T})
$$

where $\mathcal{S}:=\left\{s_{i}\right\}_{i=1}^{N}$ is the set of shapes, $\mathcal{E}:=\left\{e_{i}\right\}_{i=1}^{N}$ is the set of ellipses and $\mathcal{T}: \mathcal{E} \times \mathcal{E}$ is the tree structure describing the relationships between ellipses. In our case, we use following attributes to describe each ellipse $e$ :

$$
(\alpha, \varepsilon, \kappa, \theta):=\left(\log \sqrt{4 \pi \lambda_{1} \lambda_{2}}, \frac{\lambda_{2}}{\lambda_{1}}, \frac{4 \pi \sqrt{\lambda_{1} \lambda_{2}}}{\mu_{00}}, \frac{1}{2} \arctan \frac{2 \mu_{11}}{\mu_{20}-\mu_{02}}\right)
$$

\footnotetext{
${ }^{1}$ Remark that after replacing each shape in the tree by an approximating ellipse, the inclusion relationships between shapes may be destroyed. The displayed images are obtained by superimposing ellipses on a blank background with a large-first order.
} 

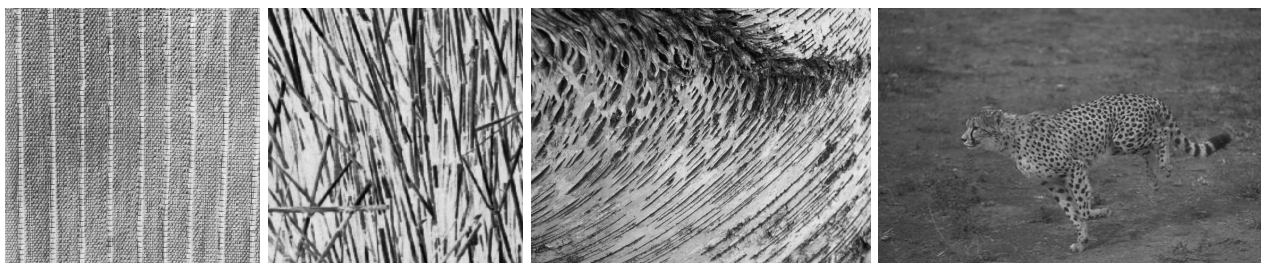

(a) Four natural texture images

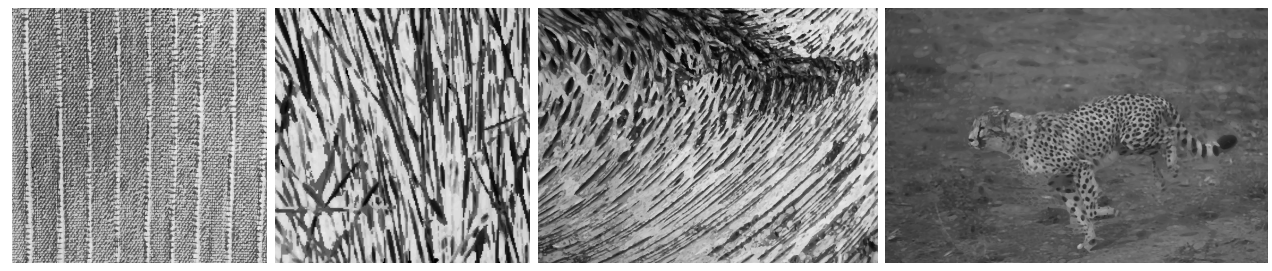

(b) The ellipse-based representation of the textures in (a).

Figure 3: Ellipse-based texture representation. Top : original texture images; Bottom : represented textures using ellipse-based textons, where each shape is replaced by an ellipse but the tree structure is kept. Refer to the text for more details.

where $\alpha, \varepsilon, \kappa$ and $\theta$ are respectively defined as log-size, elongation, compactness and orientation of the ellipses, $\mu_{p q}$ is the $(p+q)$-order moment and $\lambda_{1}$ and $\lambda_{2}$ (with $\lambda_{1} \geq \lambda_{2}$ ) are the two eigenvalues of the inertia matrix of the corresponding shape $s$.

Other important texture cues are of course contained in the tree structure. according to the relationships between ellipses. We take these into account by considering local statistics on the tree. More precisely, we use the scale ratio $\gamma$, the ratio between the area of an ellipse and the area of its parent on the tree, which is similar to [四].


and the above features only describe the geometric aspects of textures, here we make use of the contrast information by considering the normalized gray-level value $\rho$ of pixels in each ellipse. More precisely, for each pixel in an ellipse, its gray level value is normalized by the average and standard derivation of all the pixels inside this ellipse.

\section{Segmentation by grouping ellipses with active contours}

Partitioning an image into different regions of homogeneous texture with active contours has been widely studied $[\square, \square, \square]$. Here, we make use of it to identify the boundaries between different texture regions. First, in order to obtain local texture features, we cast the model in Section 2.2 to each pixel of the image, which implies that a pixel $x$ from the image $u$ is described by the features of $e(x)$, the smallest ellipse on the tree containing the pixel $^{2}$. Thus, a vector $v=(\alpha, \varepsilon, \kappa, \theta, \gamma, \rho)$ of length 6 is attached to each pixel $x$. The segmentation then amounts to partition the resulting vectorial image. We chose to use an active contour model based on the Kullback-Leibler (KL) divergence to make a 2-phase partition of the image into the background and the objects of interest. 
A recent active contour model has been proposed in [ㅁ]] to find, within an image, two regions with two probability density functions (PDFs) of texture features as disjoint as possible. We chose to adapt this scheme to our framework because it is adapted to histogram-based texture characterization and efficient. Suppose for the moment that each pixel of the image is characterized be a texture feature $f$. Let $p_{\text {in }}$ be the inside PDF, $p_{\text {out }}$ the outside PDF, $C:=C_{\text {in }}$ be the evolving region and $\Omega / C:=C_{\text {out }}$ its complementary set in $\Omega$. The method of []] suggests to maximize the KL-divergence between the PDFs of the regions inside and outside the evolving active contour $C$. The PDF corresponds to the random variable made of the texture feature $f$. The $p_{\text {in }}$ and $p_{\text {out }}$ associated with a region $C$ are evaluated thanks to a Parzen window as

$$
p_{\text {in }}(f, C)=\frac{1}{|C|} \int_{C} G_{\sigma}(f-f(x)) d x
$$

and

$$
p_{\text {out }}(f, C)=\frac{1}{|\Omega / C|} \int_{\Omega / C} G_{\sigma}(f-f(x)) d x,
$$

where $|\cdot|$ is the area of a region and $G_{\sigma}(\cdot)$ is a Gaussian kernel with zero-mean and standard deviation $\sigma$, which controls the smoothness of the approximation. The symmetric KLdivergence between $p_{\text {in }}$ and $p_{\text {out }}$ is defined as

$$
K L\left(p_{\text {in }}(f, C) \| p_{\text {out }}(f, C)\right)=\int_{-\infty}^{\infty}\left(p_{\text {in }}(f, C) \cdot \frac{p_{\text {in }}(f, C)}{p_{\text {out }}(f, C)}+p_{\text {out }}(f, C) \cdot \frac{p_{\text {out }}(f, C)}{p_{\text {in }}(f, C)}\right) d f .
$$

The segmentation then consists in maximizing the difference between the PDFs inside and outside a contour $C$, as

$$
\arg \min _{C}\left\{L(C)-\lambda K L\left(p_{\text {in }}(f, C) \| p_{\text {out }}(f, C)\right)\right\},
$$

where $L(C)$ is the length of the contour, and $\lambda$ is a regularization parameter. After computing the shape derivative, Bresson et al. [四] showed that the minimization of the energy in Equation (4) can be solved by a variational model, enabling the fast computation of a global optimum.

In our case, according to the texture modeling in Section 2.2 and making an assumption that the components of $v=(\alpha, \varepsilon, \kappa, \theta, \gamma, \rho)$ are independent, together with the additive property of KL-divergence on independent variables, we have,

$$
K L\left(p_{\text {in }}(v, C) \| p_{\text {out }}(v, C)\right)=\sum_{v \in\{\alpha, \varepsilon, \kappa, \theta, \gamma, \rho\}} K L\left(p_{\text {in }}(v, C) \| p_{\text {out }}(v, C)\right),
$$

and

$$
\arg \min _{C}\left\{L(C)-\lambda \sum_{v \in\{\alpha, \varepsilon, \kappa, \theta, \gamma, \rho\}} K L\left(p_{\text {in }}(v, C) \| p_{\text {out }}(v, C)\right)\right\},
$$

whose minimization is achieved by the variational method proposed in [ब].

\section{Experimental Results}

In this section, several examples of the resulting segmentation scheme are displayed. Figure 4 shows segmentation results on several composite texture images. Each image is composed of two different textures, which have been radiometrically corrected in order to share 

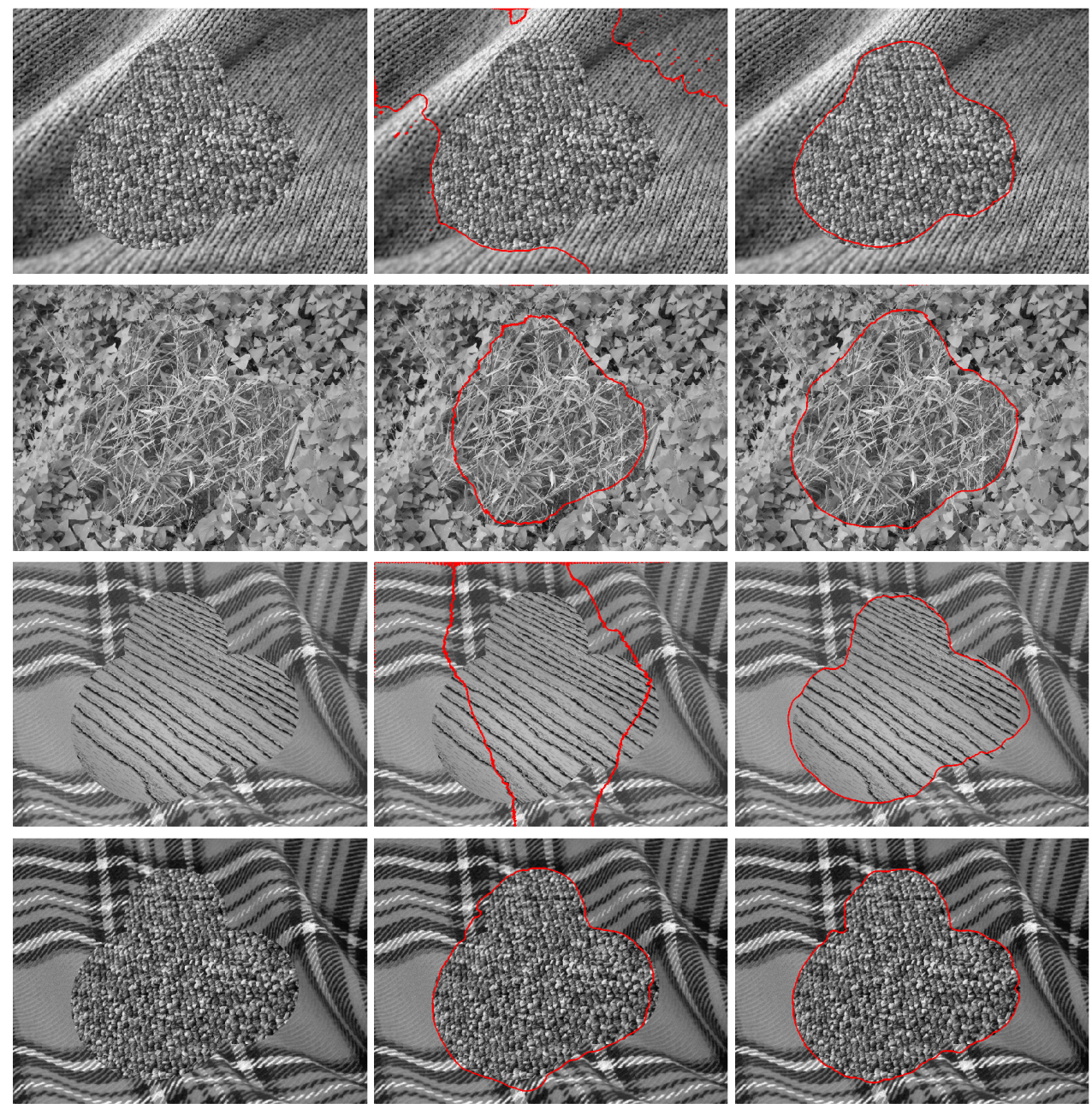

Figure 4: Segmentations of composite texture images made of two textures, radiometrically normalized to share the same mean and standard derivation. The segmentation boundaries (in red) overlay the original images. Left column: original textures; Middle column: the segmentations obtained by the method of Houhou et al. [ㅁ] ]. Right column: the segmentations obtained by the proposed method.

the same global mean and standard deviation. To compare with the state-of-the-art results, we also show the segmentation results obtained on these images using the texture features based on shape operators proposed by Houhou et al [ㄴ]], which are reported to outperform other methods, such as the model of Savig et al. [ $[\mathbf{U}]$ using the vectorial Chan-Vese model and an edge detector function based on Gabor responses. These features heavily rely on contrast information, and therefore may fail in cases where both textures share the same mean and variance. In comparison, we are able to correctly discriminate between both regions.

We also experiment on several natural images. The best segmentation results obtained with this method are shown on Figure 5. By "best", we refer to the best choice for the reg- 
ularization parameter $\lambda$ in Equation (5)). This parameter has been fixed for all experiments. Moreover, observe that the texton extraction step involves no parameter.

Figure 6 illustrates the segmentation results of two Julesz textures, which are composed of simple shapes or terminators. The segmentations are satisfying. However, observe that the obtained segmentations are inconsistent with human texture perception, since these two textures are not distinguishable by pre-attentive vision []].

Although this approach may yield excellent results, it is important to notice that those results highly depend on the regularization parameter $\lambda$ in the energy, as it is usual with active contour models and other energy minimization based segmentation models. These results could certainly benefit from recent developments in global minimization for active contour models such as those of [Q].

\section{Conclusion}

In this paper, we proposed a new texture segmentation approach by relying on the granulometrylike texrture analysis method. where ellipses are regarded as texture elements and textures are characterized by statistics from the ellipse ensembles. We argue that the boundaries between two natural texture regions can be identified well by grouping these ellipse ensembles according to some statistical properties with an active contour model. In general, this work somehow fills the gap between granulometry and texton theory on segmentation. It is convinced by the experimental results that the proposed texture segmentation approach is efficient for texture segmentation and is also available for textures with geometric transformations. Notice that this method is very related to the work of Lazebnik et al. [ $\square$ ], who used ellipse-shaped regions extracted by interest points/regions detectors to form a sparse texture representation. But they did not consider the geometric properties of the regions and did not take into account the relationships between them for texture analysis.

Acknowledgement The authors like to express their thanks to, Yann Gousseau and Julie Delon for their discussions and suggestions on this work, Nawal Houhou for kindly sharing his codes on texture segmentation, and the anonymous reviewers for their helpful comments.

\section{References}

[1] Narendra Ahuja and Sinisa Todorovic. Extracting texels in 2.1d natural textures. In Proc. of ICCV, 2007.

[2] J.-F. Aujol, G. Aubert, and L. Blanc-Féraud. Wavelet-based level set evolution for classification of textured images. IEEE Trans. Image Processing, 12:1634-1641, 2003.

[3] J. Beck. Similarity grouping of curves. Percept Mot Skills., 36(3):1331-1341, 1973.

[4] J. Beck, K. Prazdny, and A. Rosenfeld. A theory of textural segmentation. In Human and Machine Vision, pages 1-38, 1983.

[5] X. Bresson, S. Esedoglu, P. Vandergheynst, J. P. Thiran, and S. Osher. Fast global minimization of the active contour/snake model. J. Math. Imaging Vis., 28(2):151$167,2007$. 

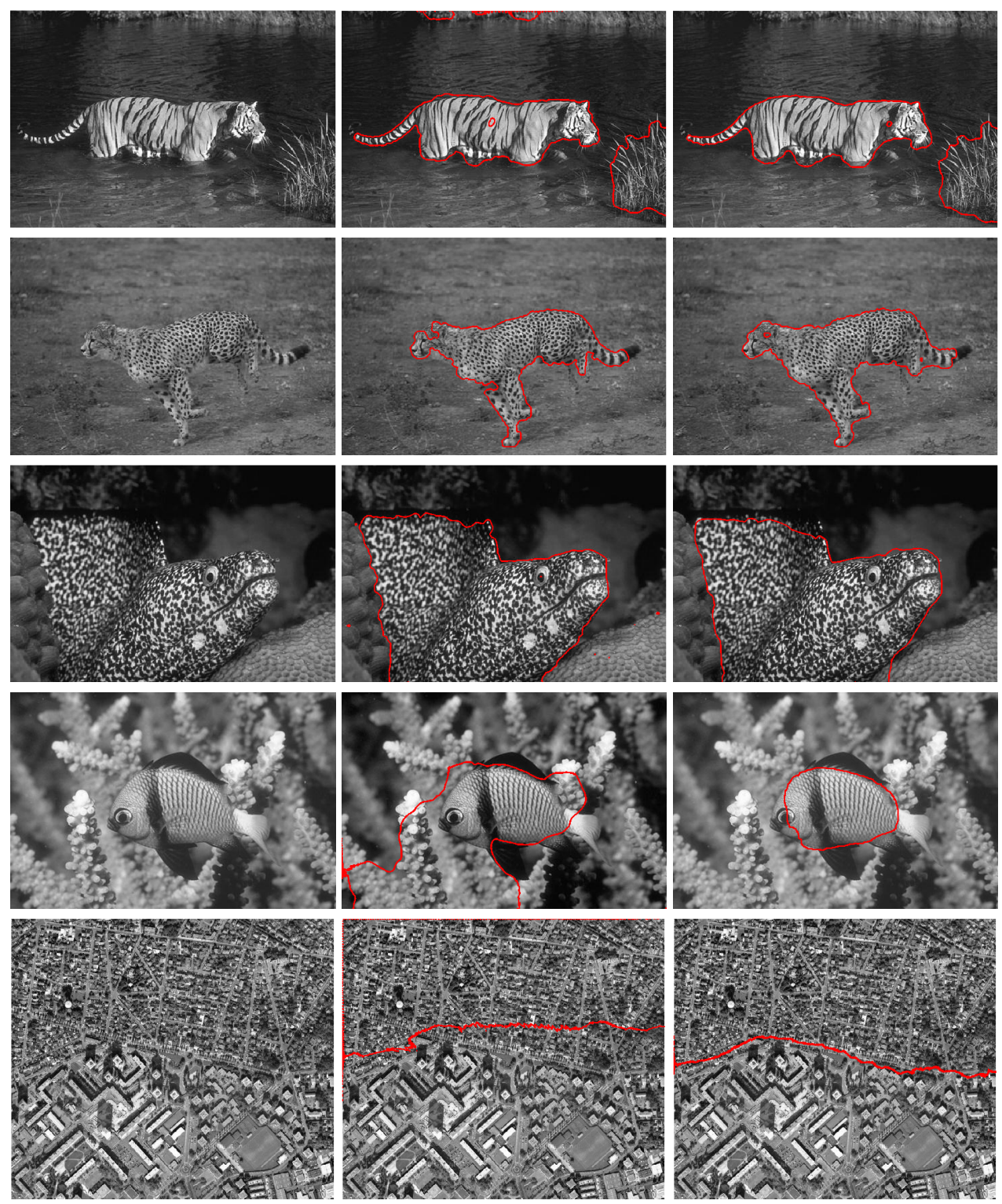

Figure 5: Segmentations of natural texture images. The segmentation boundaries overlaying the original images are displayed in red. Top row: real textures; Middle row: the segmentations obtained by the method of [ㅁ] ]. Bottom row: the segmentations obtained by the proposed method.

[6] F. Cao, P. Musé, and F. Sur. Extracting meaningful curves from images. J. Math. Imaging Vis., 22(2-3):159-181, 2005.

[7] V. Caselles, B. Coll, and J.-M. Morel. Topographic maps and local contrast changes in natural images. Int. J. Comput. Vision, 33(1):5-27, 1999. 


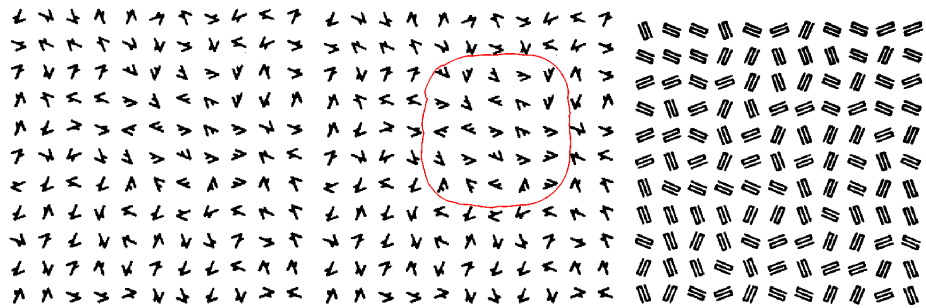

$\|\leqslant \leqslant\| \leqslant \Leftrightarrow \| \leqslant 2$ $s \leqslant s\|\| \mathbb{M}\|\leqslant\| \leqslant$

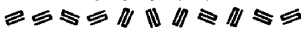

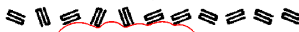

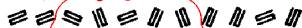
$5 \mathbb{N}=\|\geqslant\|=\|\geqslant\|$



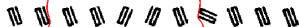

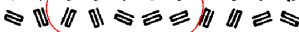

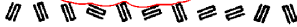

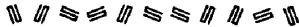

Figure 6: Experiments on the segmentation of two synthetic Julesz textures. The segmentation boundaries overlaying the original images are displayed in red. The segmentations are satisfying. However, observe that the obtained segmentations in the middle and on the right are inconsistent with human texture perception, since these two textures are not distinguishable by pre-attentive vision [ष]].

[8] V. Caselles, J.-L. Lisani, J.-M. Morel, and G. Sapiro. Shape preserving local histogram modification. IEEE Trans. Image Processing, 8(2):220-230, 1999.

[9] V. Caselles, B. Coll, and J.-M. Morel. Geometry and color in natural images. J. Math. Imaging Vis., 16(2):89-105, 2002.

[10] Y. Chen and E. R. Dougherty. Gray-scale morphological granulometric texture classification. Optical Eng., 33(8):2713-2722, 1994.

[11] N. D. Fletcher and A. N. Evans. Texture segmentation using area morphology local granulometries. In Mathematical Morphology: 40 years on. Proceedings of the 7th International Symposium on Mathematical Morphology, pages 367-376, 2005.

[12] Y. Gousseau. Texture synthesis through level sets. In Proc. of International Workshop on Texture Analysis and Synthesis, pages 53-57, Copenhagen, 2002.

[13] H. M. Hamdan and L. M. Larson. Texture classification through level lines. In Proc. of International Conference on Image Processing, pages 937-940, 2002.

[14] N. Houhou, J. P. Thiran, and X. Bresson. Fast texture segmentation model based on the shape operator and active contour. In Proc. of Computer Vision and Pattern Recognition, 2008.

[15] B. Julesz. Textons, the elements of texture perception, and their interactions. Nature, 290(5802):91-97, 1981.

[16] S. Lazebnik, C. Schmid, and J. Ponce. A sparse texture representation using local affine regions. IEEE Trans. Pattern Anal. Mach. Intell., 27(8):1265-1278, 2005.

[17] J. Malik, S.J. Belongie, T. Leung, and J.B. Shi. Contour and texture analysis for image segmentation. Int. J. Comput. Vision, 43(1):7-27, June 2001.

[18] P. Maragos. Pattern spectrum and multiscale shape representation. IEEE Trans. Pattern Anal. Mach. Intell., 11(7):701-716, 1989.

[19] P. Monasse and F. Guichard. Fast computation of a contrast invariant image representation. IEEE Trans. Image Processing, 9(5):860-872, 2000. 
[20] C. Sagiv, N. A. Sochen, and Y. Y. Zeevi. Integrated active contours for texture segmentation. IEEE Trans. Image Processing, 1:1-19, 2004.

[21] J. Serra. Image Analysis and Mathematical Morphology. Academic Press, 1982.

[22] S. Todorovic and N. Ahuja. Texel-based texture segmentation. In Proc. of International Conference on Computer Vision, pages 841-848, 2009.

[23] M. Tuceryan. Moment-based texture segmentation. In Proc. of ICPR, pages III:45-48, 1992.

[24] G.-S. Xia, J. Delon, and Y. Gousseau. Shape-based invariant texture indexing. Int. J. Comput. Vision, 88:382-403, 2010.

[25] S.-C. Zhu, C.-E. Guo, Y.-Z. Wang, and Z. Xu. What are textons? Int. J. Comput. Vision, 62(1-2):121-143, 2005. 